\title{
Design and Development of Fire Gridding Platform Based on Internet of Things
}

\author{
San-Xi WEI1, a, Jin-Chao XIAO'1,2and Ji-Hai LIU1 \\ ${ }^{1}$ Shenyang Institute of Automation, Guangzhou, Chinese Academy of Sciences, Guangzhou, China, 511458 \\ ${ }^{2}$ Shenyang Institute of Automation, Chinese Academy of Sciences, Shenyang, China, 110016 \\ asan-Xi WEl: weisanxi@gz.sia.cn
}

\begin{abstract}
This article describes the construction background about fire gridding platform, reviews the research and progress in fire Internet of Things and fire gridding. The platform includes perception / execution layer, field control layer, network layer, center platform layer and application layer, which provide a good bonding about site control and remote monitoring. This article supplies a detail design for the main functions of application and task flow of fire hazard investigation. At the same time, a digital management platform was developed.
\end{abstract}

\section{Introduction}

In recent years, Chinese economic development rapidly, industrialization, urbanization and modernization are all in a rapid progress. The explosion of various types of Industrial and commercial units in rural towns and communities increasing the pressure of fire prevention and control. The number of deaths in rural towns and communities related to fire was accounted for over $70 \%$ of the nation. In response to the severe fire safety situation, the State Council issued the "The opinions of state council on strengthening and improving the fire control work" in 2011 year. Opinions point out "the township people's governments and sub district offices must establish fire safety organization, confirm a person responsible for the fire service, push fire gridding management, enhance fire safety infrastructure, to promote the level of rural and community's fire-fighting work." In 2012, the Central Comprehensive Management of Public Security, Ministry of Public Security, the State Administration for Industry and Commerce, Ministry of Civil Affairs and the State Administration issued the "guidance about implement fire safety gridding management on villages and towns". Guidance clearly stipulate through full use of all kinds of technical protection facilities, Internet of Things (IOT) technologies and fire safety video surveillance, to achieve real-time monitoring and dynamic management for fire safety.

At present, there have same studies about fire Internet of Things and fire safety management. Wei Wang[1] explored the foundation of application of IOT in social fire safety management, presented the unsolved problems of application of IOT in social fire safety management, including the key technology of fire
Internet of Things, the special technical standard, construction and operation and so on. Qi-Yao YANG, Quan-BoGE [2]analyzed the shortcoming of city firefighting system. Based on the 3-tier structure of IOT, they proposed a smart wireless firefighting system and introduced the functions and connections within every part of the smart fire, including fire information perception, fire equipment management and the key technology. Hui-Ping HANG[3] employed complex events processing engine integrating with My Eclipse to develop a prototype system. As a result, the entire process in the system including data stream processing from IOT, events and rules modeling and on-line discrimination of false fire reporting.

Now, most studies on fire Internet of Things and grid-based of fire safety are still in the prototype stage of exploration or theoretical exploration, a small number of studies applied to practice. Zhen-Kun WU[4] designs fire safety grid-based management system framework, which is composed of base layer, sense layer, network layer, platform layer and application layer. The fire safety grid-based management realized normalized running in Anhui. At the same time, city fire control facing the difficulties such as large range of fire hazards, high environmental complexity, big fire risk, high growth disaster factor and so on. Fire control work not only collect and process large amounts of information, but also put forward higher requirements for real-time detection and real-time processing. The platform starting from small area's fire information collection, through fire real-time detection[5], fire local handling and large 
areas of grid-based management, to achieve networked and intelligent for fire prevention and control.

\section{Platform Architecture}

Platform architecture proceed from IOT's three-tier architecture: perception layer, network layer and application layer, combined with real-time fire detection [6], real-time field control technical support and various basic services. Platform architecture include the perception/Execution Layer, control hierarchy layer, network layer, center platform layer and application layer, as shown in the Figure 1.

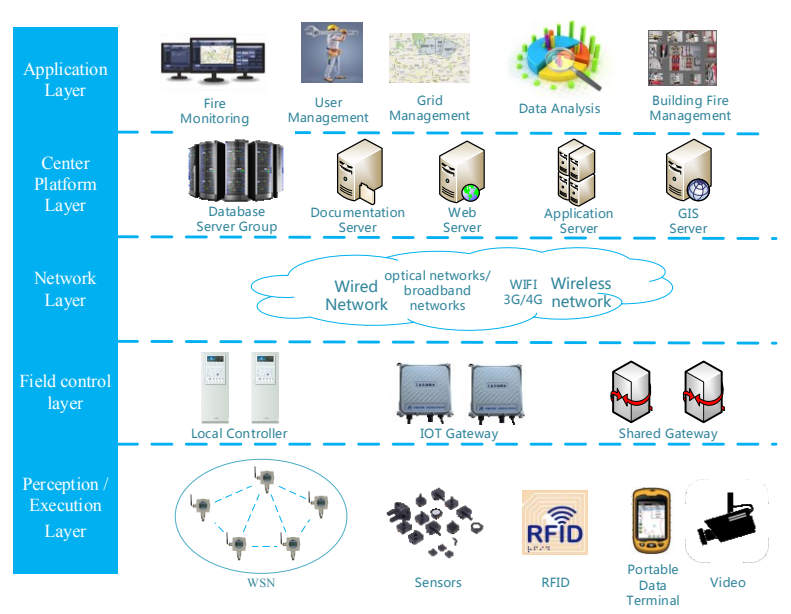

Figure 1. Platform Architecture

\subsection{Perception / Execution layer}

Perception / Execution layer including wireless sensor networks (WSN), fire sensors, video capture devices, mobile collection devices and various types of fire equipment.

Perception / Execution layer both as fire data gather agency, and as executive agency for commands from upon layers. Fire data includes the data gathered by collection equipment and person. The video surveillance equipment, temperature sensors, pressure sensors and other online perception devices constitute the main part of device data acquisition. Device data first centralized tolocal gateway and transmitted to the fire intelligence controller in field or network core of platform. Platform achieve real-time remote status monitoring of key unit's fire data by online device data acquisition. Data acquisition by personnel is complementary approaches for data acquisition, through grid inspector import the fire information by handheld devices, to achieve full coverage information collection. At the same time, the unit grid have the responsibility for perform the task of fire investigation and emergency reporting work. After the discovery of fire hazard or fire, the unit grid inspector reporting timely by handheld terminal. Control command including the commands form local fire intelligent controller and the platform. Firefighting equipment as the execution terminal for control command, realize the remote control.

\subsection{Field control layer}

Field control layer is mainly composed of fire intelligent controller and integrated gateways.

This layer plays a connecting role in the platform, including underlying devices connection and information exchange with cloud platform. Gateway collect fire equipment and fire status information to platform. Field fire intelligent controller automatically determine whether the disaster occurred and the severity of fire, make the corresponding treatment measure.

Field fire intelligent controller monitor fire alarm controller from different manufacturers and the real-time fire data; perform data frame analytical and send the monitoring information to fire control center. Controller also includes intelligence analysis function for monitoring information. Through the fire detection technology, realize real-time diagnosis for fire information. When the fire broke out, controller timely report the fire situation and location information, perform on-site emergency treatment, to promote platform field processing capacity. Controller also have the function that receive and execute commands from fire monitoring center.

Intelligent Integrated gateway contains network communication and intelligent management features. The gateway have flexible protocol processing module to support the processing of different communication protocols. Data interface section have the analytical capabilities of different application data and unified data description to support information interoperability among various network equipment. The gateway have wireless and wireline emergency switching function.

\subsection{Network layer}

Network layer composed by wireless and wired communication networks. Kinds of wired and wireless sensing devices, gateway devices, mobile terminals, grid nodes in perception layer tightly connect to platform center through network layer, achieve real-time interoperability and bidirectional flow of information. Mobile devices access platform communication center through 3G / 4G / GPRS wireless communication network. Office nodes of small grid, medium grid and large grid access platform communication center through wired network. For ensure information security, there have firewall between public network and system communication center, at the same time, the corresponding data connection in a encrypted way. Server rooms of fire brigade and fire detachments place the communication center and database of platform. Highspeed LAN connect all the point. Platform set the communication center as a pivot, through information exchange and messaging mechanism to achieve real-time and reliable flow of information between internal departments, external devices and nodes. 


\subsection{Central platform layer}

Center platform layer mainly composed by database server cluster, application servers and web servers and so on.

For large size, wide source, complex structure and heterogeneous fire data, using cluster application and distributed file systems technology to implement the cloud storage. The cloud resource management is the core of the cloud platform management .Through using performance prediction model and load forecasting model for dynamic and optimal design of resources, to achieve the smooth layer server management, storage management and network management. Resources include storage resources, computing resources and network resources.

For massive data mining of the cloud platform services layer, using distributed parallel data mining algorithms to forming high-value data such as fire management, fire prevention and control of data, emergency response data, security assessment data, etc.

\subsection{Application Layer}

Application layer mainly composed by the application functions oriented to various types of users, who associated with the fire grid management.

Application layer functions including statistical analysis, work instructions, process hazards, fire prevention, fire protection plans, risk review, task supervision, fire inspection, information collection, event handling and user management. Through the fire grid management and GIS map applications, users can view, manage, review and evaluation the fire safety unit area of jurisdiction, buildings and other hazard information. Platform establish the association a link between fire data, such the basis information of fire safety units, inspection records, hazard rectification records, fire attribute information (indoor fire facilities, floor plans, fire exits, etc.), as platform basis business data to achieve the fire safety supervision and management purpose.

\section{Design of System Function}

\subsection{Data Collection and Management}

Platform's data objects including fire safety basic data and real-time data [7].Fire safety basic data contains the GIS data, grid data and unit security data. GIS data include spatial data of different levels of the grid and spatial information of firefighting facilities within the grid.Grid cell data including attribute data of each grid, the task information and daily inspection data, etc.Unit security data including unit's basic information and fire safety information corresponding to grid cell. Fire safety real time data including real-time data collected by smoke sensors, gas sensors, temperature sensors and video surveillance. Based on "fire cloud" and cloud technology -like virtualization, distributed computing and storage, platform provide statistics, analysis and intelligent decision cloud computing support for a variety of fire data.

\subsection{Gridding and Management}

Based on existing urban geographic information systems, using spatial information gridding method, according to combining the principle of geographical division, functional division and administrative division, platform divides urban space into large, medium and small grid. Large grid corresponding to municipal districts. Medium grid corresponding to villages, towns and street community.Small grid corresponding to the administrative villages. Small grid refined into grid cell, the grid cell corresponding to village, residential, commercial area, District factories, mines and other basic spatial units.

Platform's grid management functions include grid information management, grid responsibilities management and fire unit relationships topology management. Grid management functions through the information entry, space dividing, relationships topology map and other functions to manage the information of grid and fire unit. Platform build topological relationship between large, medium and small grids, as well as topological relationship between grids with police station, fire brigade and other functional departments. The topological relations take a foundation for processes accurate, fast and efficient transfer of fire management task.

\subsection{User and Permission Management}

Platform uses topology rules between grids to achieve user management, namely achieve the differentiation and communication between large, medium, small grid and grid cell through the rule. After ensure the differentiation and communication between grids, grid users unique within the platform can to be sure. Platform provides search, interact, license, access control and other functions for users, achieves the interaction between a large number of grid users, users and services as well as users and resources. Platform achieves permission management by giving different users different functions conferred authority.

\subsection{Fire Hazard Investigation Task Management}

Platform sets module for fire hazard investigation. According to fire inspection laws, such as quarterly for large grid, monthly for medium grid, weekly for small grid, platform automatically generates inspection tasks for different grid users and design the different check table styles. Large grid and medium grid members release temporary and specific inspection tasks. Platform designs upload module for unit grid members, function of module including quickly and easily information entering, realtime pictures and voice notes sending, log automatically saving and uploading in real time. 


\subsection{Fire Awareness Training and Plans Management}

According to requirement of fire prevention, platform sets fire awareness training and plans management modules. The first module used to manage the fire publicity training in large, medium and small grids, including generating awareness training mission, carrying out fire lectures and organizing evacuation training, at the same time, save and upload training records. The second module according the actual situation, design various types of fire emergency plans to deal with all types of emergencies of fire, reduce casualties and property losses. Plans aim at specific equipment, facilities, location and environment, on the basis on safety evaluation, made scientific, effective plans and arrangements for emergency rescue agencies and person, emergency rescue equipment, facilities, conditions and environment, procedures and programs of action, methods and procedures of accident control.

\section{Process Design of Fire Hazard Investigation Task}

Fire hazard including the various potential unsafe factors for cause fire or increase the fire harm. Fire safety management is the main task of the fire gridding platform, which around the fire hazard. In addition to effective control of fire hazards, fire safety management handle the fire caused by unsafe factors that without timely and effective control. Platform considering all kinds of fire hazard, such as illegal building that fireproof rank is not up to standard, electricity using for lack of fire safety measures, fire safety spacing do not match the requirements, fire road occupied and fire equipment is not complete. Platform automatically generates inspection tasks or large grid member regularly publish special inspection tasks for fire hazard. Unit grid members execute the task. The task execution flow as figure 2 . In addition to receiving a task from large grid member, medium grid member also can publish regular fire inspection tasks to small grid member.

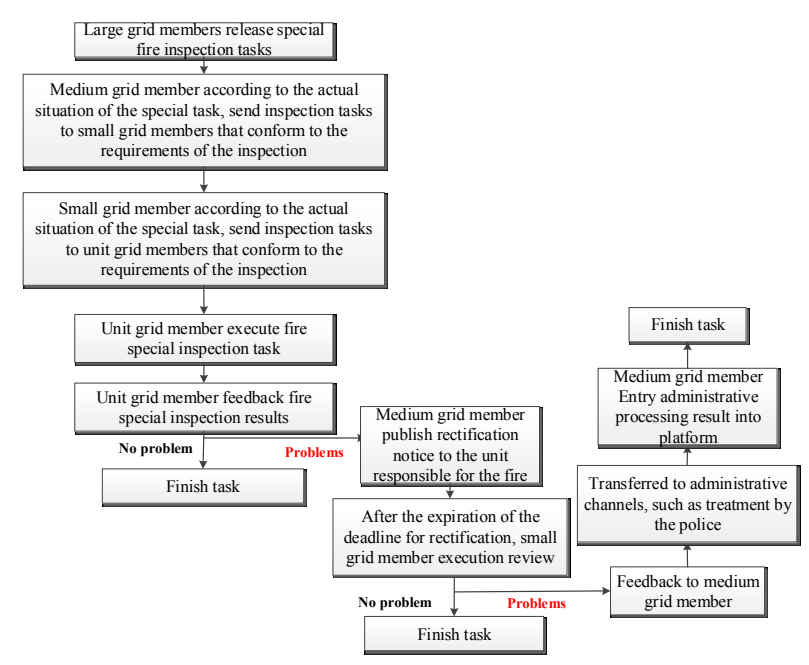

Figure 2. Task Execution Flow Diagram

\section{Application Example}

Platform uses SSH [8] software technology framework to building flexible, easily extensible and maintainable multi-tier Web applications. The SSH framework's persistence layer using Hibernate O / R mapping tools, containers using Spring Framework and the presentation layer uses Spring MVC framework. Platform's software technology architecture as Figure3,

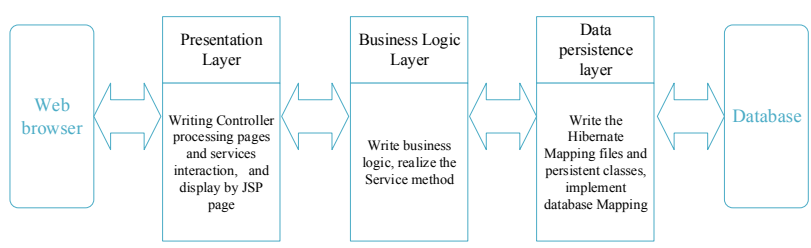

Figure 3.Software Technology Architecture

Platform management interface as figure 4,
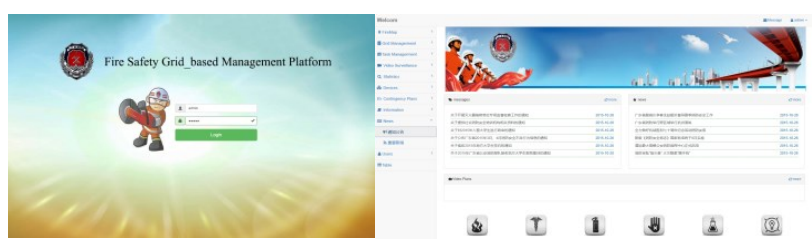

Figure 4.Platform Management Interface

\section{Summary}

This paper proposes a fire gridding platform based on the Internet of Things. Platform constructed by perception / execution layer, field control layer, network layer, platform layer center and application layer. At the same time, there have detailed analysis on each layer. Platform realizes comprehensive information perception, the smallest unit of site control, multiple forms of network transmission, coverage for core service and application extensions for the actual needs. Platform provides a full coverage, visualization, intelligent grid management to fire authorities, effectively promote the improvement of the level of fire safety management.

\section{Acknowledgement}

Supported Project: National 863 plans projects (2014AA041801-1).

\section{References}

1. Wei WANG, Jing-Li NAN and Jian-Wei BO. Fire Science and Technology, 31, 864-866(2012).

2. Qi-Yao YANG, Quan-Bo GE. Fire Science and Technology, 33: 552-554(2014)

3. Hui-Ping HANG. Master's Thesis, Zhejiang University of Science and Technology, 2015. 
4. Zhen-Kun WU, Hao HU, Tao WENG, Li-Wei PAN and Peng MEI. Fire Science and Technology, 34, 260-262(2015)

5. Jia WANG. Master's Thesis, Changchun University of Science and Technology. 2012.

6. Qing-Feng ZHOU, Xuan-Fang YANG and Xiang-Yi ZHAN. Fire Science and Technology, 34, 16411644(2015)
7. Yong-Xuan FANG, Bin-Bin LU, Li-Wei PAN and Peng MEI, China Public Security, Academy Edition, 35, 28-31(2014)

8. Xiu-Juan HUANG Computer Knowledge and Technology,11,63-65(2015). 